\title{
Electromagnetic Analysis of Beam- Scanning Antenna at Millimeter-Wave Band Based on Photoconductivity Using Fresnel-Zone-Plate Technique
}

\author{
M. Hajian, G. A. de Vree, and L. P. Ligthart \\ IRCTR/TU Delft \\ Mekelweg 42600 GA Delft, The Netherlands \\ Tel: +31 15 2786256; Fax: +31 152784046; E-mail: m.hajian@its.tudelft.nl
}

\begin{abstract}
The proposed antenna is a Fresnel-zone-plate antenna (FZPA) antenna in the Ka band that scans the beam of a millimeterwave source. The antenna includes a semiconductor wafer (silicon, gallium arsenide) in which a spatially varying density of charge carriers is selectively established through selective optical illumination using a pulse laser. It is shown that the beam of the antenna can be scanned through the space by reconfiguring the masking of the wafer. The beam scanning is done in the transmission mode. The analysis of the far-zone radiation characteristics is presented using Physical Optics. Results for the antenna gain, the efficiency, and the co- and cross-polar patterns are given.
\end{abstract}

Keywords: Beam steering; scanning antennas; millimeter wave antennas; photoconductivity; Fresnel zone plate antennas; masks; antenna radiation patterns; polarization

\section{Introduction}

\begin{abstract}
Tn the past two years, extensive research has been carried out at IRCTR (International Research Centre for Telecommunicationtransmission and Radar) at the Technical University in Delft, The Netherlands, in order to analyze and model the reflection and transmission coefficients of a semiconductor, i.e., doped silicon, under illumination of light [1-2]. It was found that the characteristics of a semiconductor material can be substantially changed once it is illuminated with light. The preliminary measurements, in a closed waveguide structure, show that there is a total reflection of millimeter-wave (MMW) and microwave (MW) power after the laser is turned on. The laser light causes a considerable change in the equivalent conductivity of the silicon wafer, changing it from an insulator to a good conductor.
\end{abstract}

This property of the semiconductor material can be used to realize an antenna that can form and scan the beam of a millimeterwave source. This paper gives the preliminary theoretical results on how such a property can be used to scan the beam based on the FZP (Fresnel-zone-plate) technique. The idea behind the Fresnel zone plate antenna (FZPA) is a well known concept, and it has been the topic of the research in many papers [3-6]. Its advantages are a flat configuration, conformability, and low cost. The disadvantage is its low efficiency. Hristov, Herben, and Leyten first analyzed the vectorial far field of the Fresnel-zone-plate antenna [4-5]. The analysis also covered the scanning performance of a Fresnel-zone-plate antenna using an offset feed, based on
Kirchhoff diffraction theory. The aim of this paper is to demonstrate the beam scanning of a Fresnel-zone-plate antenna using the photoconductivity concept. The antenna includes a semiconductor wafer, a light source, and a millimeter-wave source. The source is placed at the focal point of the Fresnel-zone-plate antenna. Beam scanning is achieved by masking the semiconductor wafer adaptively, using the light source. The part of the wafer that is illuminated by the light behaves more or less like a good conductor, and the wafer can thereby reflect or transmit the incident millimeterwave radiation. The change in the semiconductor's properties is caused by carrier generation using the light source. Since the optical masking can be reconfigured rapidly (due to the short carrier lifetime of the semiconductor material), the beam can be scanned in space. Note that this paper does not cover the optical part of the antenna. It is assumed that the projection of the Fresnel zone plate on the wafer has already been done. The antenna is used in the transmission mode. The analysis is done at $\mathrm{Ka}$ band $(35 \mathrm{GHz})$.

\section{Preliminary Measurements on a Silicon Wafer}

A silicon wafer, with a thickness of $0.3 \mathrm{~mm}$, was bounded by two media in a closed waveguide structure. Two detectors were used to measure the reflection and transmission of the microwave and millimeter-wave source. A mirror and a lens were used to focus the laser light into the waveguide [2]. Complete reflection 
occurred after the laser was turned on. An on/off ratio of $20 \mathrm{~dB}$ was measured, and a carrier lifetime of $6 \mu \mathrm{s}$ was measured. There was good similarity between the behavior of the theoretical and the measured results. The measurement results reported in [2] showed that in the reflection mode, after the laser was turned on, the electromagnetic waves losses were of the order of $0.01 \mathrm{~dB}$. For the transmission mode, the losses depended on the coating of the semiconductor material. More research is needed for measuring and analyzing the losses of the semiconductor material in the transmission mode. In the past, Woerdman [7] has experimentally generated a hologram on the silicon with a resolution of the order of $10 \mu \mathrm{m}$. This resolution is sharp enough to realize antenna ele- ments on the semiconductor material for beam scanning.

A laser pulse with an energy of $1 \mathrm{~mJ} / \mathrm{cm}^{2}$ and a duration of $10 \mu \mathrm{s}$ was used for the measurements. The power of the pulse laser is given by $P=\frac{\text { Energy }}{\text { Tijd }}=\frac{1 \mathrm{~mJ} / \mathrm{cm}^{2}}{10 \mathrm{~ms}}=100 \mathrm{~W} / \mathrm{cm}^{2}$. This means that for a wafer with area of $400 \mathrm{~cm}^{2}$, an optical power of $40 \mathrm{~kW}$ is needed. In our measurements, it was shown that for a wafer with a thickness of $0.3 \mathrm{~mm}$, only $30 \%$ of the power was needed to produce complete reflection. This means that an optical laser with a power of $12 \mathrm{~kW}$ satisfied the requirements. For a wafer with a radius of $10 \mathrm{~cm}$ (used in this paper), an optical laser with a power of $\approx 31.5 \mathrm{~kW}$ is needed. Note that this power is calculated as if the design illuminated the complete wafer. However, this is not the case, since for the beam-scanning Fresnel-zone-plate antenna, almost half of the area is used in both the reflection and transmission modes. It can be concluded that the antenna can be realized at $\mathrm{Ka}$ band with $\approx 16 \mathrm{~kW}$ of laser power.

\section{Fresnel-Zone-Plate Antenna}

Figure 1 shows the Fresnel-zone-plate antenna configuration. The Fresnel zone plate acts as a lens-like focusing element [8-12]. It concentrates an incident plane wave to the focal point. In the transmitting mode, it converts a spherical wave into a plane wave.

As is shown in Figure 1, the antenna is divided into zones, with a zone number $m$. The even zones ( $m$ is even) are transparent and the odd zones are blocked, or the other way around. Per the definition, the distance, $\rho$, from the feed to a point $(x, y)$ in the $m$ th zone obeys the following inequality:

$$
\frac{m \lambda}{2}+F \leq \rho \leq \frac{(m+1) \lambda}{2}+F
$$

where $\lambda$ is the wavelength and $F$ is the focal distance. $\rho$ is the distance from the feed to a point on the plate, given by $\rho=\sqrt{F^{2}+x^{2}+y^{2}}$, or, in polar coordinates, $\rho=\sqrt{F^{2}+r^{2}}$.

On the $m$ th border, the following equation holds for $b_{m}$ :

$$
\sqrt{F^{2}+b_{m}^{2}}=\frac{m \lambda}{2}+F
$$

From Equation (2), the radius of the zone is given by

$$
b_{m}=\sqrt{\lambda m\left(F+\frac{\lambda m}{4}\right)}
$$

\section{Geometry of Antenna}

Projecting an elliptical Fresnel-zone-plate pattern with varying shape onto the wafer can scan the beam of the antenna. This configuration is used in this paper since the transmission Fresnelzone-plate antenna does not suffer from blocking by the feed.

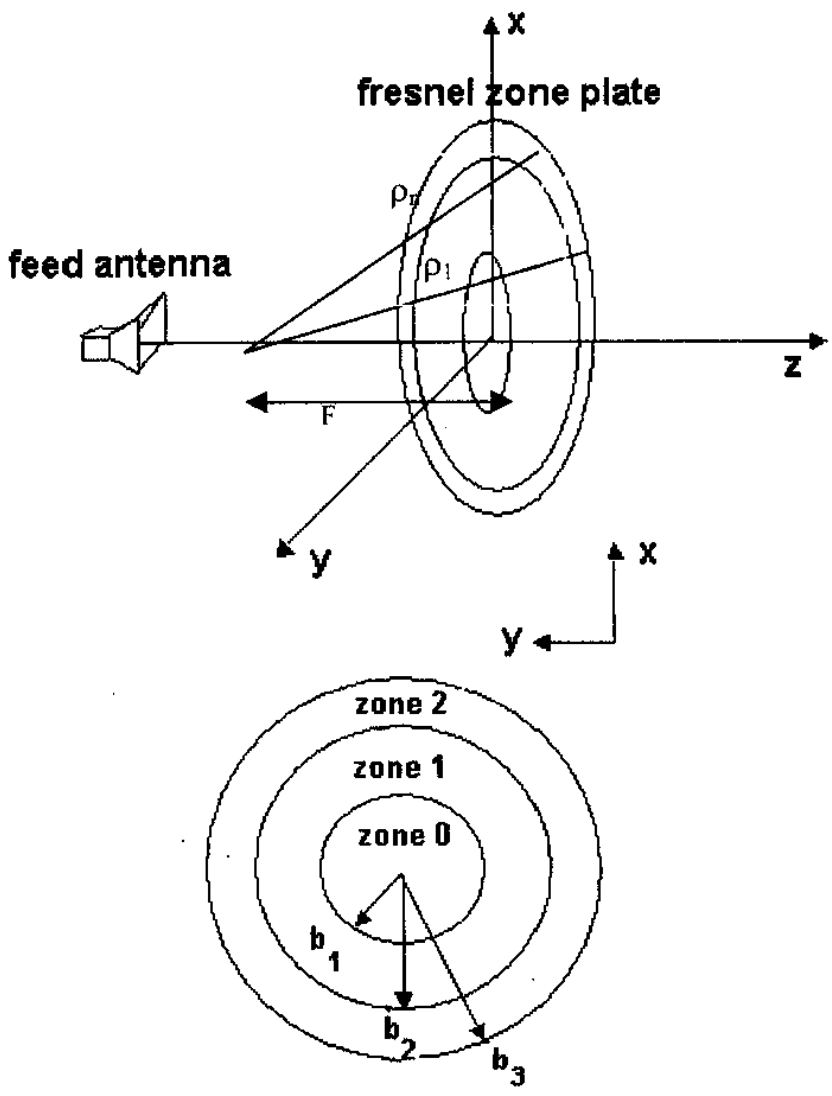

front view of FZP

Figure 1. The antenna configuration for a focused Fresnelzone-plate antenna.

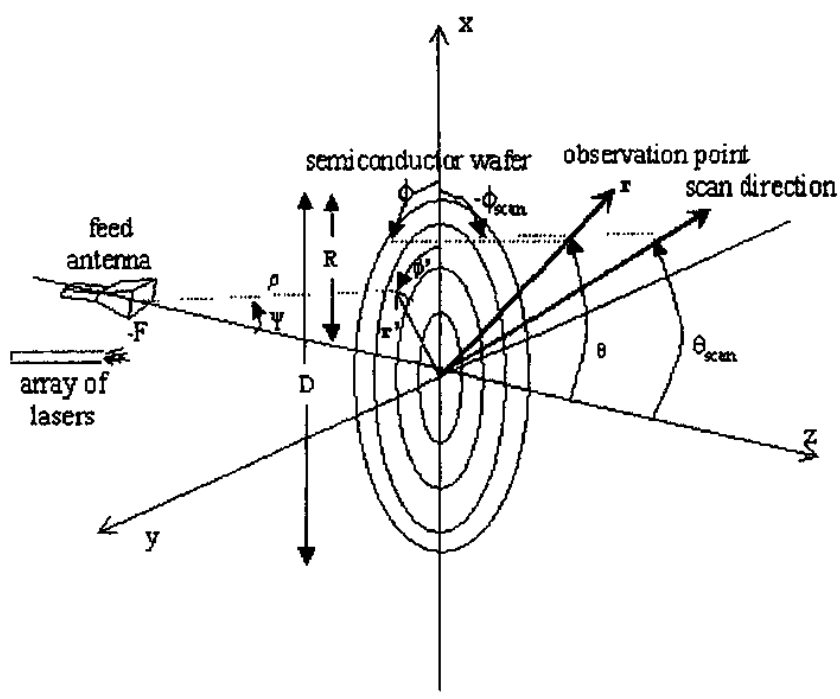

Figure 2. The geometry of the antenna. 


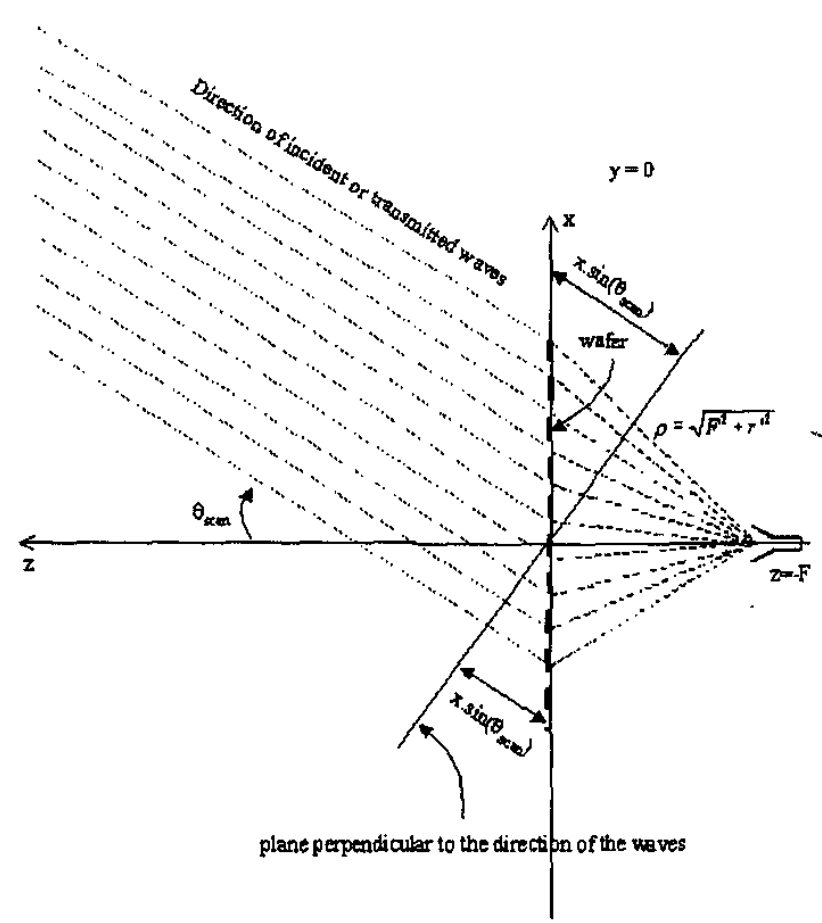

Figure 3. A cross section of the antenna configuration at $y=0$ for $\theta_{\text {scan }} \neq 0^{\circ}, \phi_{\text {scan }}=0^{\circ}$.

Figure 2 shows the geometry of the antenna and the coordinate system. The vector $\mathbf{r}$ indicates the far-field observation point with spherical coordinates $(r, \theta, \phi)$. A point on the wafer is defined by the vector $\mathbf{r}^{\prime}$. The spherical coordinates $\left(\rho, \psi, \phi^{\prime}\right)$ are used to describe the radiation pattern of the feed. The scan direction is indicated by $\theta_{\text {scan }}$ and $\phi_{\text {scan }}$. The wafer is circular and lies in the $x-y$ plane, and the wafer's dimension is given by its radius, $R$, or its diameter, $D$. The feed is placed at the focal distance, $F$, from the wafer.

\section{Masking the Wafer}

Projecting an elliptical Fresnel-zone-plate pattern with varying shape onto the wafer can scan the beam of the antenna. To determine which parts of the wafer need to be illuminated in order to scan the beam, the principle of the Fresnel-zone-plate antenna is used. The masking to scan the beam in the elevation plane $\left(\phi_{\text {scan }}=0^{\circ}\right)$ is first derived. The design will then be extended to scan the beam in the azimuth plane $\left(\phi_{s c a n} \neq 0^{\circ}\right)$ by simply rotating the masking $\phi_{s c a n}$. The zones of a Fresnel-zone-plate antenna, with maximum radiation in the $+z$ direction $\left(\phi_{\text {scan }}=0^{\circ}\right)$, are defined by Equation (1). When $\theta_{\text {scan }}$ is not zero, Equation (1) has to be modified to account for oblique incidence or transmission. To illustrate this, the cross section of the configuration for $y=0$ is shown in Figure 3.

From Figure 3, it can be seen that path length needs to be corrected by $x \sin \left(\theta_{\text {scan }}\right)$ to scan the beam to $(\theta, \phi)=\left(\theta_{\text {scan }}, 0^{\circ}\right)$. Therefore, Equation ( 1 ) is rewritten as

$$
\frac{m \lambda}{2}+F \leq \sqrt{r^{2}+F^{2}}-x \sin \left(\theta_{\text {scan }}\right) \leq \frac{(m+1) \lambda}{2}+F .
$$

The zone number, $m$, for every point $\left(r^{\prime}, \phi^{\prime}\right)$ on the wafer can be calculated from Equation (4). This gives the following expression for $m$ :

$$
m=\left\lfloor 2 \frac{\sqrt{r^{\prime 2}+F^{2}}-r^{\prime} \cos \left(\phi^{\prime}\right) \sin \left(\theta_{\text {scan }}\right)-F}{\lambda}\right\rfloor,
$$

where the substitution $x=r^{\prime} \cos \left(\phi^{\prime}\right)$ is used. In order to scan the beam in the azimuthal plane, the parameter $\phi_{\text {scan }}$ is added to rotate the masking. In this case, Equation (5) becomes

$$
m=\left\{2 \frac{\sqrt{r^{\prime 2}+F^{2}}-r^{\prime} \cos \left(\phi^{\prime}-\phi_{\text {scan }}\right) \sin \left(\theta_{\text {scan }}\right)-F}{\lambda}\right] .
$$

In this paper, the masking that is used blocks the odd zones ( $m$ is odd), and lets the even zones be transparent. Hence, the masking function, which returns one if a point $\left(r^{\prime}, \phi^{\prime}\right)$ lies on an open zone and zero if it lies a the blocked zone, is given by

$$
\begin{aligned}
& \operatorname{mask}\left(r^{\prime}, \phi^{\prime}, F, \theta_{\text {scan }}, \phi_{\text {scan }}, \lambda\right) \\
= & I s E v e n\left(\left\lfloor 2 \frac{\sqrt{r^{\prime 2}+F^{2}}-r^{\prime} \cos \left(\phi^{\prime}-\phi_{\text {scan }}\right) \sin \left(\theta_{\text {scan }}\right)-F}{\lambda}\right\rfloor\right)
\end{aligned}
$$

The function IsEven is a binary function. It returns a one if its parameter is even, and zero if it is odd.

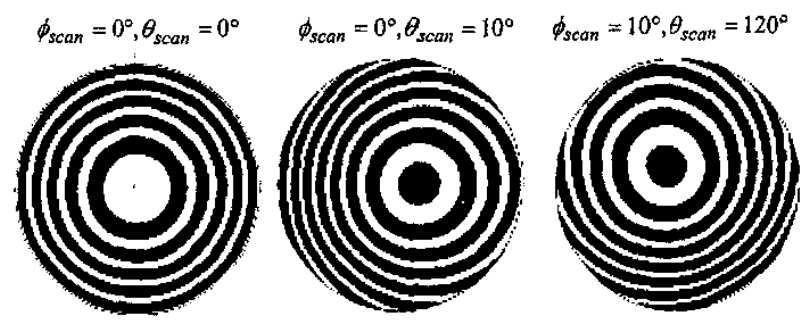

Figure 4a. The wafer masking for a wafer with a diameter of $D=20 \mathrm{~cm}$ and a focal distance of $F=9 \mathrm{~cm}$.

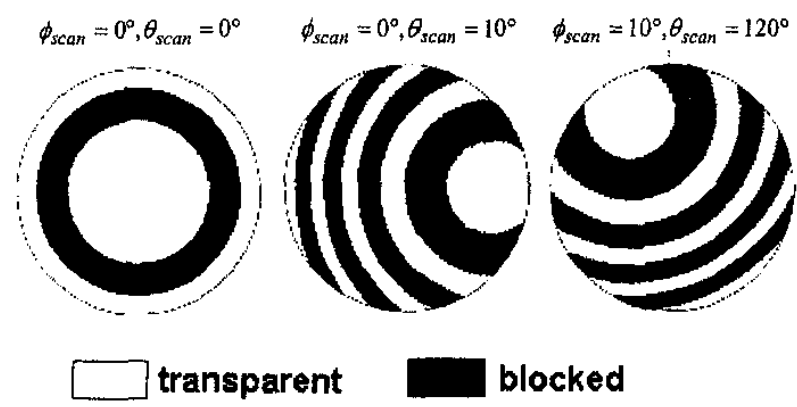

Figure $4 \mathrm{~b}$. The wafer masking for a wafer with a diameter of $D=20 \mathrm{~cm}$ and a focal distance of $F=40 \mathrm{~cm}$. 
For $\theta_{\text {scan }}=0^{\circ}$, the masking function in Equation (7) gives a circular Fresnel-zone plate masking for the wafer. If the scan direction is not normal to the wafer's surface $\left(\theta_{\text {scan }} \neq 0^{\circ}\right)$, the wafer masking becomes an elliptical Fresnel zone plate. Since the wafer has a limited size, the zones on the wafer are not always complete zones, and are truncated at the edge of the wafer. When the beam is scanned in the azimuth plane, the masking is rotated Figure 4 illustrates the masking layout for different scanning angles at $35 \mathrm{GHz}$.

\section{The Formulation of the Radiated Field}

In this section, Love's field-equivalence principle is used to derive the expression for the gain of the antenna [13]. To model the feed antenna, the following gain function is used [14]:

$$
G_{\text {feed }}(\psi)=\left\{\begin{array}{cc}
2(n+1) \cos ^{n}(\psi) & \text { if }|\psi|<\frac{\pi}{2} \\
0 & \text { if }|\psi|>\frac{\pi}{2}
\end{array},\right.
$$

where $n$ is the taper factor, and $\psi$ is the extend angle as shown in Figure 2. The incident field from the feed on the wafer is given by

$$
E_{i}(r)=e_{y} \sqrt{\frac{2 G_{f e e d}\left[\arctan \left(\frac{r^{\prime}}{F}\right), n\right] \eta P_{t}}{4 \pi\left(F^{2}+r^{\prime 2}\right)}} e^{-j k \sqrt{F^{2}+r^{\prime 2}}}
$$

where $P_{t}$ is the total power transmitted by the feed and $\eta$ is the wave impedance. It is assumed that the incident electric field is linearly $y$ polarized, and that the wafer lies in the far zone of the feed antenna. The equivalent magnetic current, $M_{s}$, is given by [13]

$$
M_{s}\left(r^{\prime}, \phi^{\prime}\right)=-2 e_{z} \times E_{i}\left(r^{\prime}, \phi^{\prime}\right)=-2 e_{x} E_{i}\left(r^{\prime}, \phi^{\prime}\right) .
$$

Note that it is assumed that the electric field in the "apertures" is equal to the incident field (the Physical Optics approximation) Another assumption is that the only contribution to the far field is from the field in the open zones of the wafer.

The electric far field of the antenna is calculated using the magnetic auxiliary function, $\mathbf{L}$. The electric auxiliary function, $\mathbf{N}$, is zero. The two components of the auxiliary function $\mathbf{L}$ are given by

$$
\begin{gathered}
L_{\theta}=\cos (\theta) \cos (\phi) \int_{0}^{2 \pi} \int_{0}^{R}\left[M_{s} \operatorname{mask}\left(r^{\prime}, \phi^{\prime}, F, \theta_{s c a n}, \phi_{s c a n}, \lambda\right)\right. \\
\left.e^{j k r^{\prime} \sin (\theta) \cos \left(\phi-\phi^{\prime}\right)} r^{\prime} d r^{\prime} d \phi^{\prime}\right]
\end{gathered}
$$

The electric far fields are related to the auxiliary function $\mathbf{L}$ as follows:

$$
E_{0}=-\frac{j k e^{-j k r}}{4 \pi r} L_{\phi}
$$

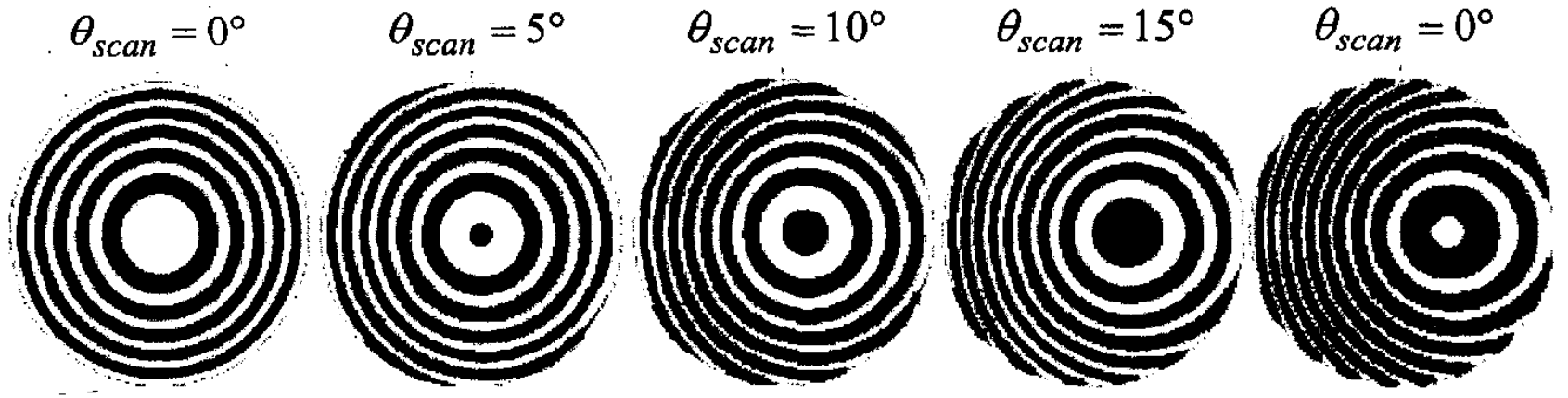

Figure 5a. The masking of the wafer for a diameter of $D=20 \mathrm{~cm}$ and a focal length of $F=9 \mathrm{~cm}$.

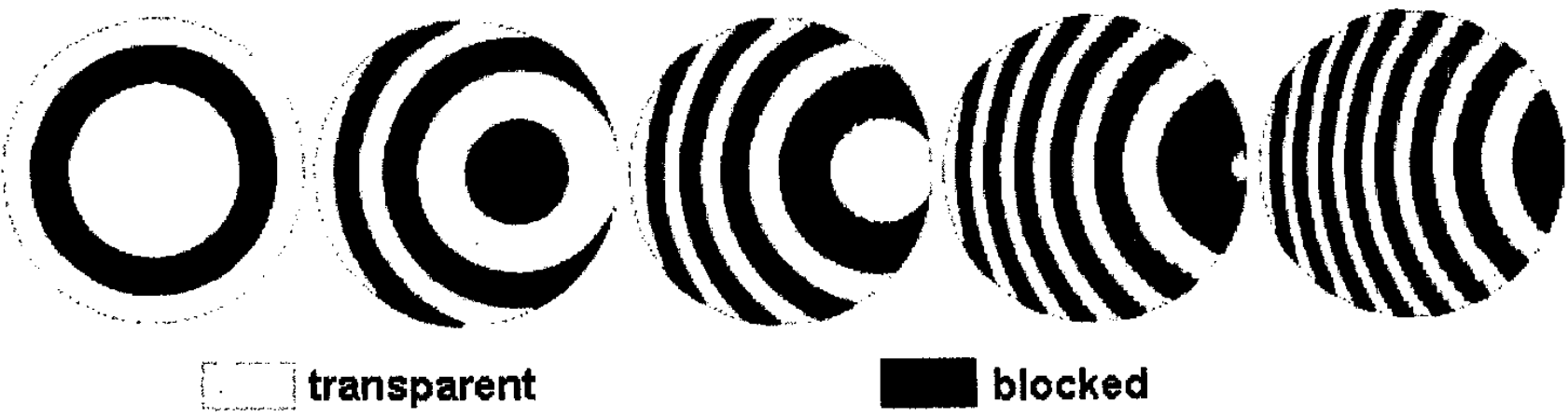

Figure 5b. The masking of the wafer for a diameter of $D=20 \mathrm{~cm}$ and a focal length of $F=40 \mathrm{~cm}$. 


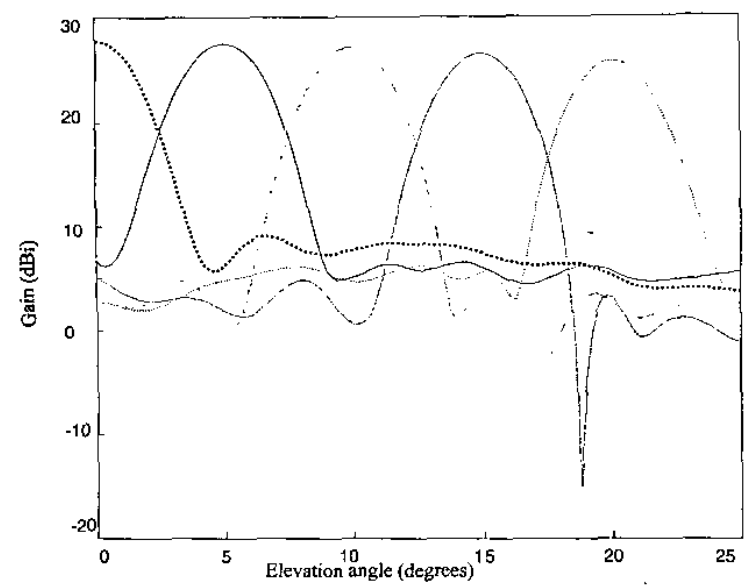

Figure 6a. The scanning performance of a size-limited Fresnelzone-plate antenna for the masking given in Figure 5 with $n=5: \cdots \theta_{\text {scan }}=0^{\circ} ;-\theta_{\text {scan }}=5^{\circ} ; \cdots \theta_{\text {scan }}=10^{\circ} ;-\cdots$ $\theta_{\text {scan }}=15^{\circ} ;-\cdots-\theta_{\text {scan }}=20^{\circ}$.

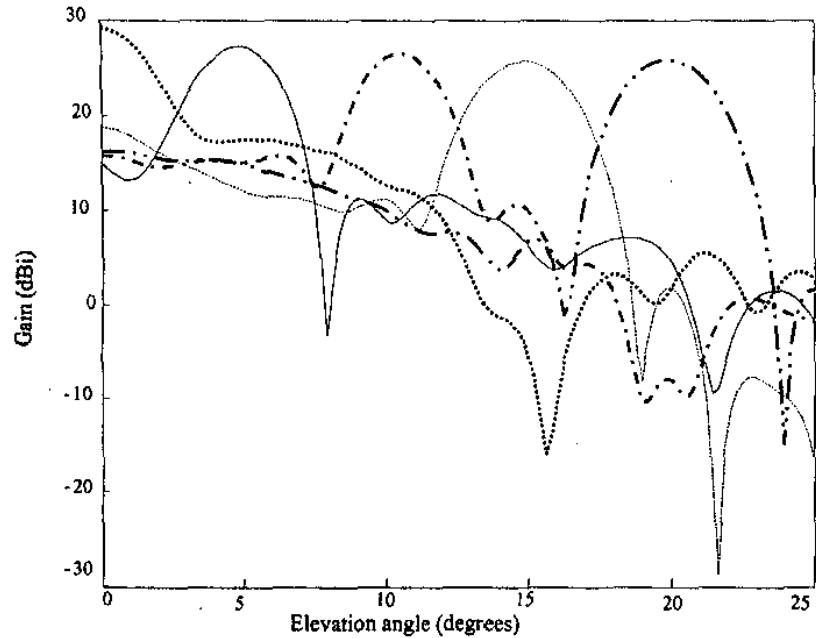

Figure $6 \mathrm{~b}$. The scanning performance of a size-limited Fresnelzone-plate antenna for the masking given in Figure 5 with $n=65: \cdots \cdots \cdots \theta_{\text {scan }}=0^{\circ} ;-\theta_{\text {scan }}=5^{\circ} ; \cdots \cdot \theta_{\text {scan }}=10^{\circ} ;---$ $\theta_{\text {scan }}=15^{\circ} ;-\cdots \theta_{\text {scan }}=20^{\circ}$.

$$
E_{\phi}=\frac{j k e^{-j k r}}{4 \pi r} L_{\theta}
$$

Using Equations (13) and (14), the gain of the antenna is given by

$$
G=\frac{2 \pi r^{2}}{\eta P_{t}}\left(\left|E_{\phi}\right|^{2}+\left|E_{\theta}\right|^{2}\right)
$$

Combining Equations (8) to (15) leads to the following expression for the gain, $G(\theta, \phi)$ :

$$
G(\theta, \phi)=\frac{2 F^{n}}{\lambda^{2}}(n+1)\left[\cos ^{2}(\theta) \cos ^{2}(\phi)+\sin ^{2}(\phi)\right] I n t
$$

where Int is given by

$$
\begin{aligned}
I n t=\mid \int_{0}^{2 \pi} \int_{0}^{R} \frac{r^{\prime} \operatorname{mask}\left(r^{\prime}, \phi^{\prime}, F, \theta_{\text {scan }}, \phi_{\text {scan }}, \lambda\right)}{\left(F^{2}+r^{\prime 2}\right)^{\frac{1}{4}(n+2)}} \\
\quad \times\left. e^{-j k\left[\sqrt{F^{2}+r^{\prime 2}}-r^{\prime} \sin (\theta) \cos \left(\phi^{\prime}-\phi\right)\right]} d r^{\prime} d \phi^{\prime}\right|^{2},
\end{aligned}
$$

where the mask function is given by Equation (7). Figure 5 shows the masking layout for a wafer with a diameter of $20 \mathrm{~cm}$ at $35 \mathrm{GHz}$ with focal distances of $9 \mathrm{~cm}$ and $40 \mathrm{~cm}$. Figure 6 shows the antenna pattern for scanning the beam up to an elevation angle of $\theta_{\text {scan }}=20^{\circ}$ using the masking given in Figure 5 .

It can be shown that rotating the masking with $\phi_{\text {scan }}$ will move the main beam to $\phi=\dot{\phi}_{\text {scan }}$ without affecting $\theta_{\text {scan }}$. Figures 7 and 8 show the masking layouts for $\theta_{\text {scan }}=15^{\circ}$, $\phi_{\text {scan }}=-45^{\circ}, \phi_{\text {scan }}=30^{\circ}$, and the radiation patterns.

\section{A Comparison of FZPA Beam Scanning Using Offset Feed and Variable Masking}

The antenna proposed in this paper scans its beam by masking the semiconductor wafer as a function of time, without moving the feed antenna. In [15], the far-field and scan performance of a

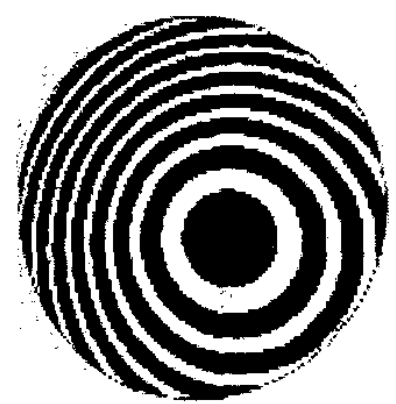

Figure 7a. The masking layout for $\theta_{\text {scan }}=15^{\circ}, \phi_{\text {scan }}=-45^{\circ}$, , $F=9 \mathrm{~cm}, n=5$.

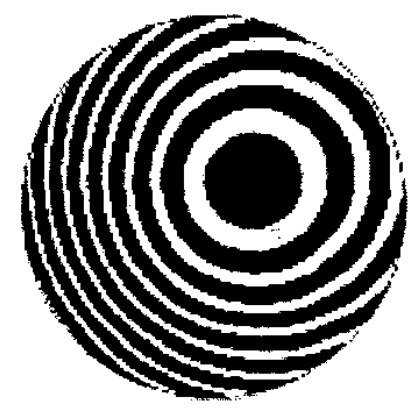

\section{transparent blocked}

Figure 7b. The masking layout for $\theta_{\text {scan }}=15^{\circ}, \phi_{\text {scan }}=30^{\circ}$, , $F=9 \mathrm{~cm}, n=5$. 


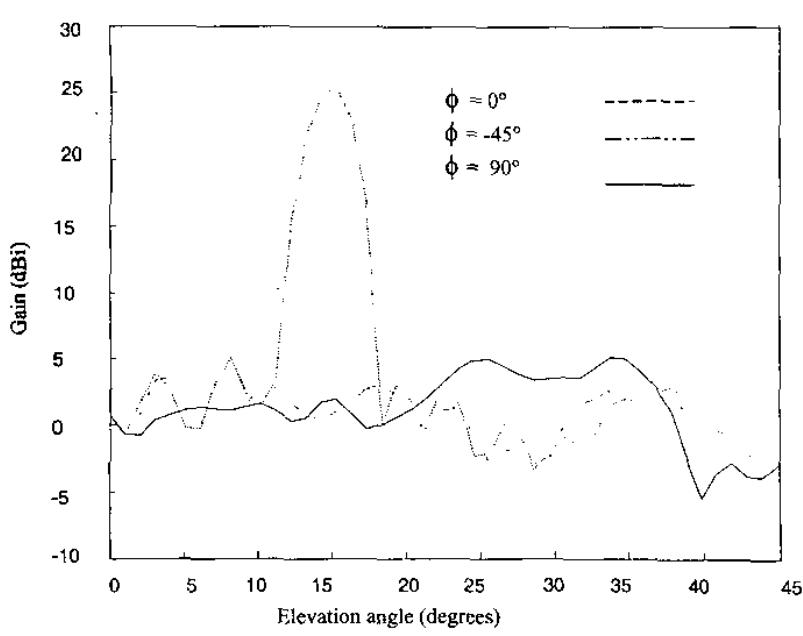

Figure 8a. Simulation results for $\theta_{\text {scan }}=15^{\circ}, \phi_{\text {scan }}=-45^{\circ}$, $F=9 \mathrm{~cm}, n=5$.

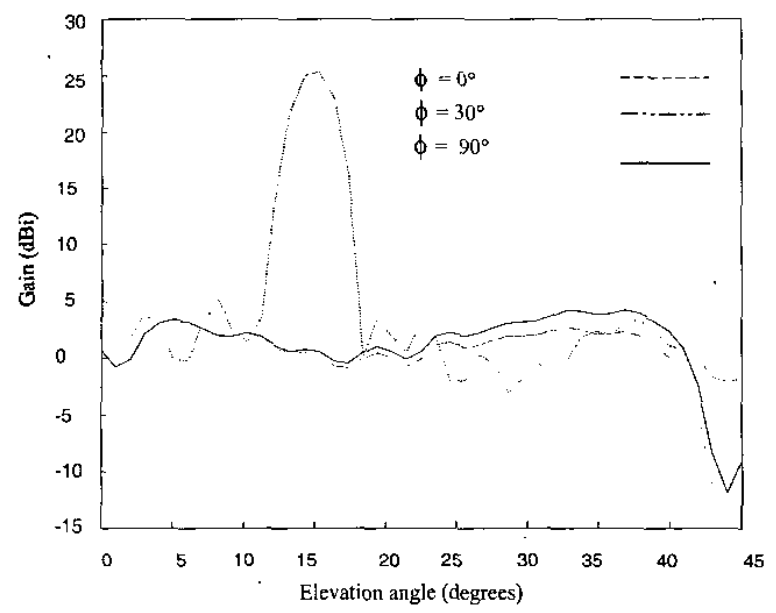

Figure 8b. Simulation results for $\theta_{\text {scan }}=15^{\circ}, \phi_{\text {scan }}=30^{\circ}$, , $F=9 \mathrm{~cm}, n=5$.

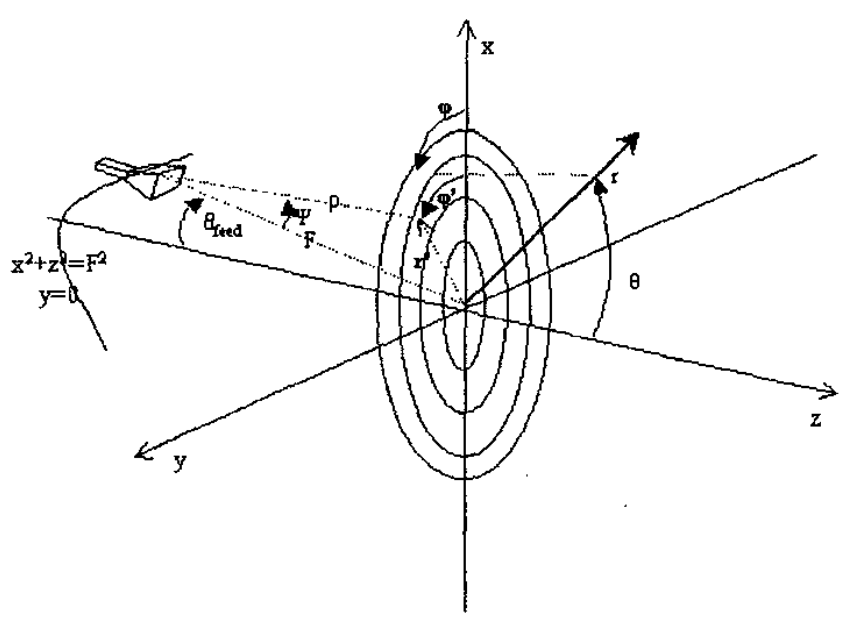

Figure 9. The geometry of the de-focused Fresnel-zone-plate antenna.

18
Fresnel-zone-plate antenna created by displacing the feed antenna is analyzed. In this section, the two configurations are compared. The comparison is made for antennas with the same dimensions operating at the same frequency as in [16]. Figure 9 shows the configuration of a defocused Fresnel-zone-plate antenna.

The difference between the geometry given in Figure 8 and the configuration in Figure 2 is that the feed in this case is displaced by an angle $\theta_{\text {feed }}$, and the Fresnel zone plate is fixed. The distance from the feed antenna to the center of the wafer is equal to the focal distance, $F$, and the feed is displaced in the $x-z$ plane. Therefore, the scan curvature of the feed antenna is described by

$$
x^{2}+z^{2}=F^{2}, \quad y=0 \text {. }
$$

The gain of the feed antenna is given by Equation (8). This gives the following expression for the incident electric field:

$$
E_{i}\left(r^{\prime}, \phi^{\prime}\right)=e_{y} \sqrt{\frac{2 \eta P_{t} G_{\text {feed }}\left[\psi\left(r^{\prime}, \phi^{\prime}\right), n\right]}{4 \pi \rho^{2}\left(r^{\prime}, \phi^{\prime}\right)}} e^{-j k \rho\left(r^{\prime}, \phi^{\prime}\right)} .
$$

$\rho\left(r^{\prime}, \phi^{\prime}\right)$ is the distance from the feed to a point $\left(r^{\prime}, \phi^{\prime}\right)$ on the Fresnel zone plate. Using Pythagoras' rule, $\rho\left(r^{\prime}, \phi^{\prime}\right)$ is found to be $\rho\left(r^{\prime}, \phi^{\prime}\right)$

$=\sqrt{\left[r^{\prime} \cos \left(\phi^{\prime}\right)-F \sin \left(\theta_{\text {feed }}\right)\right]^{2}+r^{2} \sin ^{2}\left(\phi^{\prime}\right)+F^{2} \cos ^{2}\left(\theta_{\text {feed }}\right)}$.

$\psi\left(r^{\prime}, \phi^{\prime}\right)$ is given by

$$
\psi\left(r^{\prime}, \phi^{\prime}\right)=\arccos \left[\frac{\rho\left(r^{\prime}, \phi^{\prime}\right)^{2}+F^{2}-r^{\prime 2}}{2 F \rho\left(r^{\prime}, \phi^{\prime}\right)}\right] .
$$

Using Equations (11)-(14), the total electric far field is the sum over the $M$ transparent zones:

$$
\begin{aligned}
& E_{\theta}=\sum_{m=0}^{M-1} E_{\theta, 2 m}, \\
& E_{\phi}=\sum_{m=0}^{M-1} E_{\phi, 2 m} .
\end{aligned}
$$

The gain is of the antenna is given by

$G(\theta, \phi)=\frac{2(2 F)^{-n}(n+1)}{\lambda^{2}}\left[\cos ^{2}(\theta) \cos ^{2}(\phi)+\sin ^{2}(\phi)\right]|T|^{2}$,

where $T$ is a sum over an integral:

$$
\begin{aligned}
T=\sum_{m=0}^{M-1} \int_{b(2 m)}^{b(2 m+1)} \int_{0}^{2 \pi} \frac{r^{\prime}}{\rho\left(r^{\prime}, \phi^{\prime}\right)}\left(\frac{\rho\left(r^{\prime}, \phi^{\prime}\right)^{2}+F^{2}-r^{\prime 2}}{\rho\left(r^{\prime}, \phi^{\prime}\right)}\right)^{\frac{n}{2}} \\
\times e^{-j k\left[\rho\left(r^{\prime}, \phi^{\prime}\right)-r^{\prime} \sin (\theta) \cos \left(\phi^{\prime}-\phi\right)\right]} d \phi^{\prime} d r^{\prime},
\end{aligned}
$$

where $\rho\left(r^{\prime}, \phi^{\prime}\right)$ and $b(m)$ are given by Equations (20) and (3), respectively. $M$ is the number of open Fresnel zones. The gain and

IEEE Antennas and Propagation Magazine, Vol. 45, No. 5, October 2003 


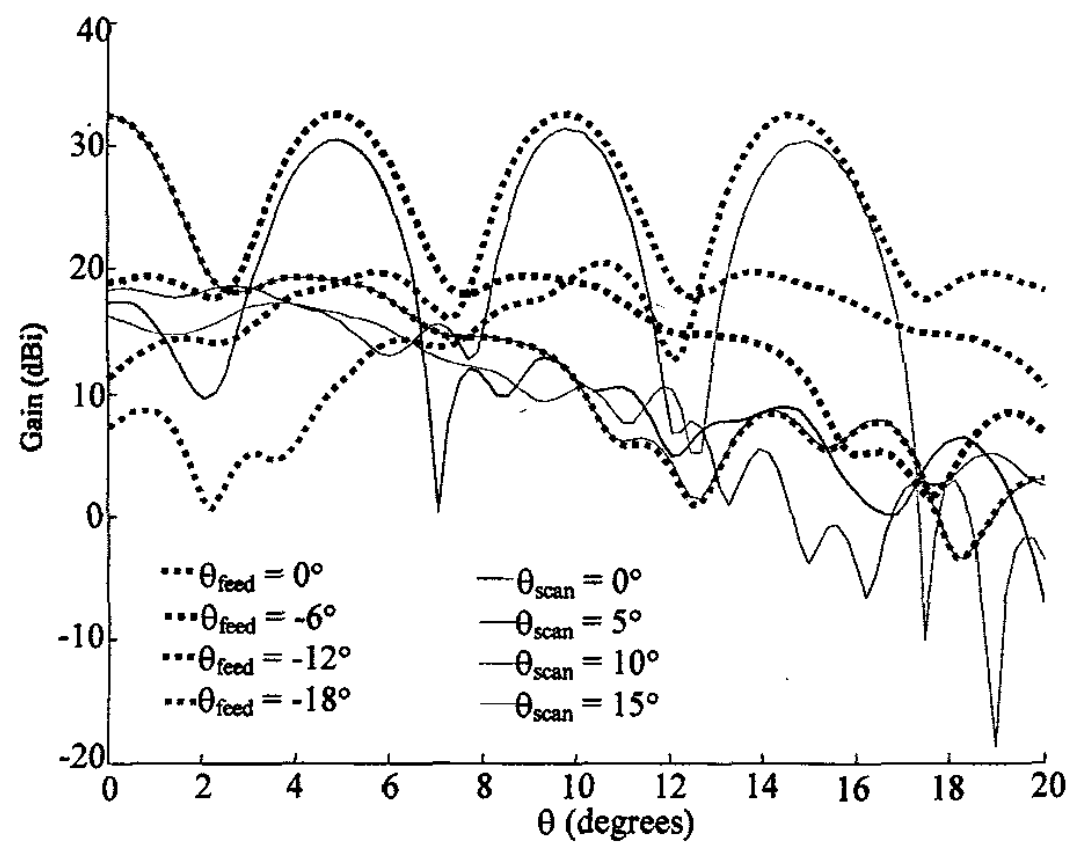

Figure 11a. The beam-scanning antenna pattern for a defocused Fresnel-zone-plate antenna (dotted lines) and a Fresnel-zone-plate antenna with variable masking (solid lines) for $F=1.88 \mathrm{~m}$.

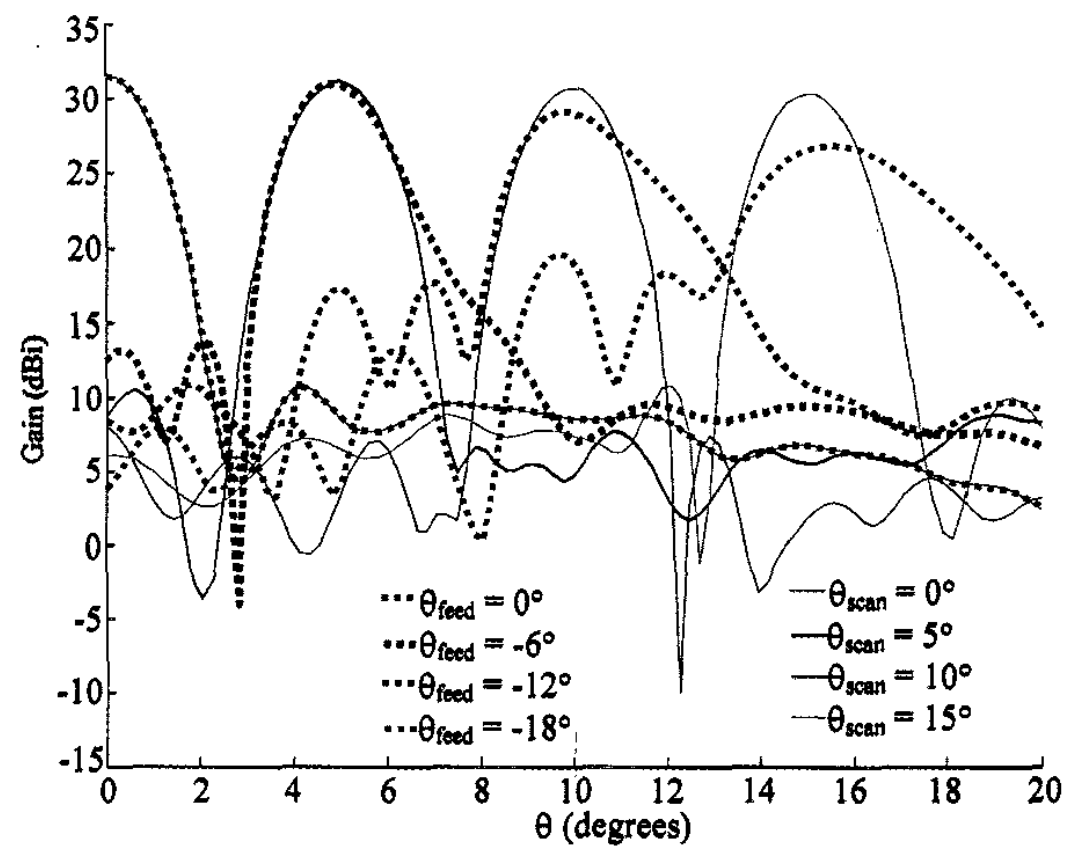

Figure 11a. The beam-scanning antenna pattern for a defocused Fresnel-zone-plate antenna (dotted lines) and a Fresnel-zone-plate antenna with variable masking (solid lines) for $F=.522 \mathrm{~m}$. 
scan performance of the defocused Fresnel-zone-plate antenna is calculated using Equations (19), (24), and (25), while Equations (16) and (17) are used for the antenna with variable Fresnel zone plate-masking. Two focal distances were used for the comparison: $F=0.522 \mathrm{~m}$ and $F=1.88 \mathrm{~m}$. The calculations were done at $11.1 \mathrm{GHz}$. The diameter of the Fresnel zone plate was chosen to be approximately one meter, such that the Fresnel zone plate had complete zones and the last zone was a transparent zone. Therefore, the relation between the radius, $R$, of the wafer and the number of the transparent zones, $M$, is given by

$$
R=b_{2 M-1}=\sqrt{2(M-1) \lambda\left[F+\frac{2 \lambda(M-1)}{4}\right]} .
$$

Figure 10 shows the masking and Fresnel zone plate structure. The simulation results for the radiation patterns are shown in Figure 11.

Comparing the results in Figure 11 reveals several differences between the two beam-scanning techniques. For a focal distance $F=1.88 \mathrm{~m}$, the defocused Fresnel-zone-plate antenna has a higher gain. At the same time, the gain hardly decreases with increasing scanning angle. For $F=0.522 \mathrm{~m}$, the antenna with variable Fresnel-zone-plate masking has a higher gain, and this decreases less than for the antenna with $F=1.88 \mathrm{~m}$. Another difference is that the main beam of the defocused Fresnel-zoneplate antenna is asymmetrical for a small focal distance. The beam of the antenna with variable Fresnel-zone-plate masking remains more symmetrical. The asymmetry and strong decrease in gain of the defocused Fresnel-zone-plate antenna can be understood as follows. If the focal distance is small compared to the size of the Fresnel zone plate, the amplitude distribution from the feed on the wafer becomes asymmetrical. This causes a decrease in gain and an asymmetrical beam.

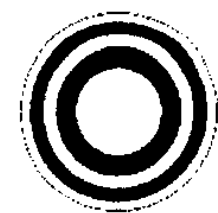

(a)

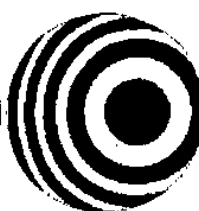

(b)

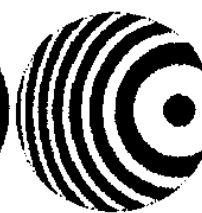

(c)

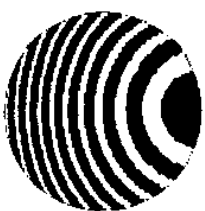

(d) transparent

blocked

Figure 10a. The masking for a de-focused Fresnel-zone-plate antenna with $F=1.88 \mathrm{~m}$ for (a) $\theta_{\text {scan }}=0^{\circ}$; (b) $\theta_{\text {scan }}=5^{\circ}$; (c) $\theta_{\text {scan }}=10^{\circ}$; (d) $\theta_{\text {scan }}=15^{\circ}$.

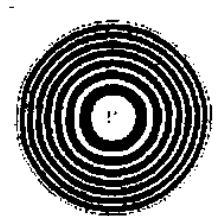

(a)

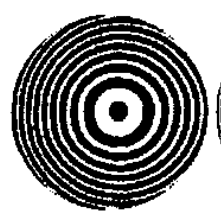

(b)

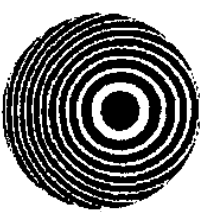

(c)

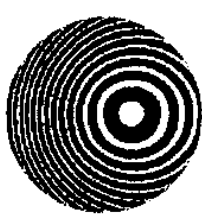

(d)

transparent

blocked

Figure 10b. The masking for a de-focused Fresnel-zone-plate antenna with $F=.522 \mathrm{~m}$ for (a) $\theta_{\text {scan }}=0^{\circ}$; (b) $\theta_{\text {scan }}=5^{\circ}$; (c) $\theta_{\text {scan }}=10^{\circ} ;$ (d) $\theta_{\text {scan }}=15^{\circ}$.

The decrease in gain of the Fresnel-zone-plate antenna with variable masking can be explained as follows. A Fresnel-zoneplate masking with a high value of focal distance has large zones. For $\theta_{\text {scan }} \approx 0^{\circ}$, a large transparent zone lies at the center of the wafer. As the scan angle, $\theta_{\text {scan }}$, increases, this is no longer the case. Since the power density is higher close to the center of the wafer, the gain decreases more with increasing scan angle for high values of focal distance.

\section{Efficiency of the Antenna}

The efficiency of the antenna is discussed in this section. The polarization efficiency is discussed separately in the next section.

\subsection{Area Efficiency}

Not all of the incident power on the wafer is effectively used. Only the area of the open zones is used. The area efficiency, $\varepsilon_{\text {area }}$, is defined as the fraction of the wafer's area that is effectively used:

$$
\begin{aligned}
& \mathcal{E}_{\text {area }}= \frac{P_{\text {transp.zones }}}{P_{\text {wafer }}} \\
&= \int_{0}^{R 2 \pi} \int_{0}^{2 \pi} P\left(r^{\prime}, \phi^{\prime}\right) \operatorname{mask}\left(r^{\prime}, \phi^{\prime}, F, \theta_{\text {scan }}, \phi_{\text {scan }}, \lambda, \text { mode }\right) r^{\prime} d \phi^{\prime} d r^{\prime} \\
& \int_{0}^{R 2 \pi} \int_{0}^{R} P\left(r^{\prime}, \phi^{\prime}\right) r^{\prime} d \phi^{\prime} d r^{\prime}
\end{aligned}
$$

\subsection{Taper Efficiency}

A uniform amplitude distribution on the wafer would produce the maximum gain. The taper efficiency, $\varepsilon_{\text {taper }}$, is a measure of the gain reduction due to a nonuniform amplitude distribution. It is given as follows:

$$
\varepsilon_{\text {taper }}=\frac{\text { actual gain }}{\text { gain if amplitude distribution is uniform }} .
$$

After some manipulation, the taper efficiency is given by [17]

$$
\begin{aligned}
& \varepsilon_{\text {taper }}=\left(F^{2}+r_{2}^{2}\right)^{\frac{1}{2}(n+2)} \\
& \times \mid \int_{0}^{R 2 \pi} \int_{0}^{2 \pi} \frac{r^{\prime} m a s k\left(r^{\prime}, \phi^{\prime}, F, \theta_{\text {scan }}, \lambda, \text { mode }\right)}{\left(F^{2}+r^{\prime 2}\right)^{(n+2) / 4}} \\
& \times\left|e^{-j k\left[\sqrt{F^{2}+r^{\prime 2}}-r^{\prime} \sin (\theta) \cos \left(\phi^{\prime}-\phi\right)\right]} d \phi^{\prime} d r^{\prime}\right|^{2} \\
& \times \mid \int_{0}^{R 2 \pi} \frac{r^{\prime} m a s k\left(r^{\prime}, \phi^{\prime}, F, \theta_{\text {scan }}, \lambda, m o d e\right)}{\left(F^{2}+\dot{r}^{\prime 2}\right)^{(n+2) / 4}} \\
& \quad \times\left. e^{-j k\left[\sqrt{F^{2}+r^{\prime 2}}-r^{\prime} \sin (\theta) \cos \left(\phi^{\prime}-\phi\right)\right]} d \phi^{\prime} d r^{\prime}\right|^{-2}
\end{aligned}
$$

IEEE Antennas and Propagation Magazine, Vol. 45, No. 5, October 2003 


\subsection{Phase Efficiency}

The phase efficiency, $\varepsilon_{\text {phase }}$, is defined as the gain reduction due to the fact that not all waves are in phase. It is defined as

$$
\varepsilon_{\text {phase }}=\frac{\text { actual gain }}{\text { gain if fields on the wafer have uniform phase }}
$$

This gives the following expression for the phase efficiency:

$$
\begin{aligned}
\varepsilon_{\text {phase }}=\mid \int_{0}^{R 2 \pi} \int_{0}^{2 \pi} \frac{r^{\prime} \text { mask }\left(r^{\prime}, \phi^{\prime}, F, \theta_{\text {scan }}, \lambda, \text { mode }\right)}{\left(F^{2}+r^{\prime 2}\right)^{(n+2) / 4}} \\
\times\left|e^{-j k\left[\sqrt{F^{2}+r^{\prime 2}}-r^{\prime} \sin (\theta) \cos \left(\phi^{\prime}-\phi\right)\right]} d \phi^{\prime} d r^{\prime}\right|^{2} \\
\times \mid \int_{0}^{R 2 \pi} \frac{r^{\prime} m a s k\left(r^{\prime}, \phi^{\prime}, F, \theta_{s c a n}, \lambda, \text { mode }\right)}{\left(F^{2}+r^{\prime 2}\right)^{(n+2) / 4}} \\
\times\left. e^{-j k\left[F-r^{\prime} \cos \left(\phi^{\prime}\right)-r^{\prime} \sin (\theta) \cos \left(\phi^{\prime}-\phi\right)\right]} d \phi^{\prime} d r^{\prime}\right|^{-2} .
\end{aligned}
$$

\subsection{Spillover Efficiency}

The spillover efficiency, $\varepsilon_{\text {spillover }}$, is the fraction of the total power radiated by the feed that is intercepted by the wafer:

$$
\begin{aligned}
\varepsilon_{\text {spillover }}= & \frac{\text { power incident on wafer }}{\text { total power transmitted by feed }} \\
= & \frac{\int_{0}^{\psi_{0}} G_{f e e d}(\psi) \sin (\psi) d \psi}{\int_{0}^{\pi} G_{f e e d}(\psi) \sin (\psi) d \psi} .
\end{aligned}
$$

Using Equation (8), the spillover efficiency is given by

$$
\varepsilon_{\text {spillover }}=1-\cos ^{n+1}\left(\psi_{0}\right),
$$

with $\psi_{0}$ being equal to $\arctan (R / F)$.

Table 1. The values used in the simulations of efficiency versus focal distance.

\begin{tabular}{|l|c|c|c|}
\hline Parameter & Description & \multicolumn{2}{|c|}{ Value } \\
\hline$\theta_{\text {scan }}$ & Elevation scan angle & $0^{\circ}, 5^{\circ}, 10^{\circ}, 15^{\circ}, 20^{\circ}$ \\
\hline$\phi_{\text {scan }}$ & Azimuth scan angle & \multicolumn{2}{|c|}{$0^{\circ}$} \\
\hline$F$ & Focal distance & $0 \ldots 40 \mathrm{~cm}$ & $0 \ldots 70 \mathrm{~cm}$ \\
\hline$n$ & Feed taper factor & 5 & 65 \\
\hline$R$ & Wafer diameter & \multicolumn{2}{|c|}{$10 \mathrm{~cm}$} \\
\hline$\lambda$ & Wavelength & $8.57 \mathrm{~mm}(35 \mathrm{GHz})$ \\
\hline
\end{tabular}

Table 2. The efficiencies for two configurations where the focal distance is optimized for a certain feed antenna.

\begin{tabular}{|c|c|c|c|c|c|}
\hline$\theta_{\text {scan }}$ & $\varepsilon_{\text {spillover }}$ & $\varepsilon_{\text {area }}$ & $\varepsilon_{\text {taper }}$ & $\varepsilon_{\text {phase }}$ & $\varepsilon_{\text {total }}$ \\
\hline \multicolumn{5}{|c|}{$n=5, F=9 \mathrm{~cm}$} \\
\hline $0^{\circ}$ & $92 \%$ & $56 \%$ & $40 \%$ & $42 \%$ & $11 \%$ \\
\hline $5^{\circ}$ & $92 \%$ & $55 \%$ & $41 \%$ & $41 \%$ & $10 \%$ \\
\hline $10^{\circ}$ & $92 \%$ & $52 \%$ & $39 \%$ & $41 \%$ & $9.5 \%$ \\
\hline $15^{\circ}$ & $92 \%$ & $48 \%$ & $37 \%$ & $41 \%$ & $8.5 \%$ \\
\hline $20^{\circ}$ & $92 \%$ & $49 \%$ & $36 \%$ & $38 \%$ & $7.9 \%$ \\
\hline \multicolumn{5}{|c|}{$n=65, F=40 \mathrm{~cm}$} \\
\hline $0^{\circ}$ & $87 \%$ & $71 \%$ & $61 \%$ & $43 \%$ & $14 \%$ \\
\hline $5^{\circ}$ & $87 \%$ & $53 \%$ & $57 \%$ & $42 \%$ & $8.6 \%$ \\
\hline $10^{\circ}$ & $87 \%$ & $49 \%$ & $56 \%$ & $40 \%$ & $7.4 \%$ \\
\hline $15^{\circ}$ & $87 \%$ & $49 \%$ & $61 \%$ & $39 \%$ & $6.2 \%$ \\
\hline $20^{\circ}$ & $87 \%$ & $50 \%$ & $60 \%$ & $40 \%$ & $6.5 \%$ \\
\hline
\end{tabular}

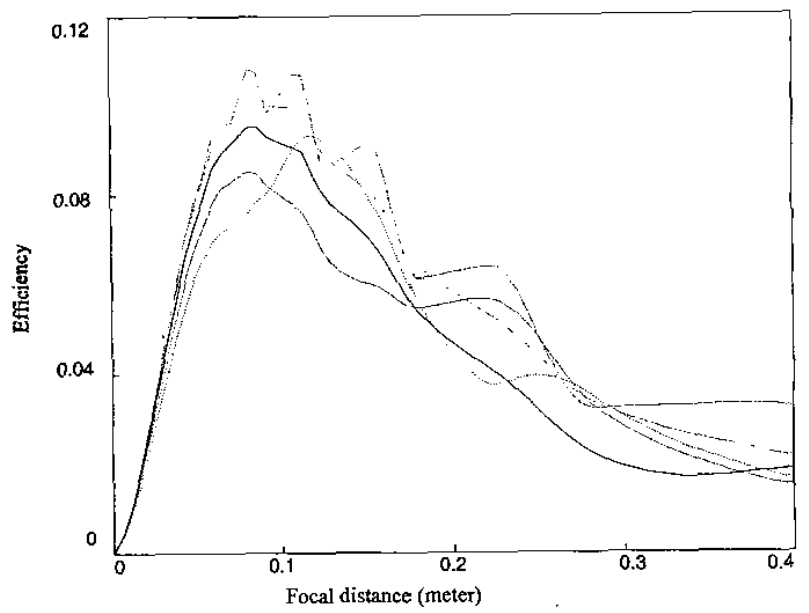

Figure 12. The total efficiency as a function of the focal distance for a feed antenna with $n=5: \cdots \theta_{\text {scan }}=0^{\circ} ; \cdots$ $\theta_{\text {scan }}=5^{\circ} ;-\theta_{\text {scan }}=10^{\circ} ;---\theta_{\text {scan }}=15^{\circ} ;-\cdots-\theta_{\text {scan }}=20^{\circ}$.

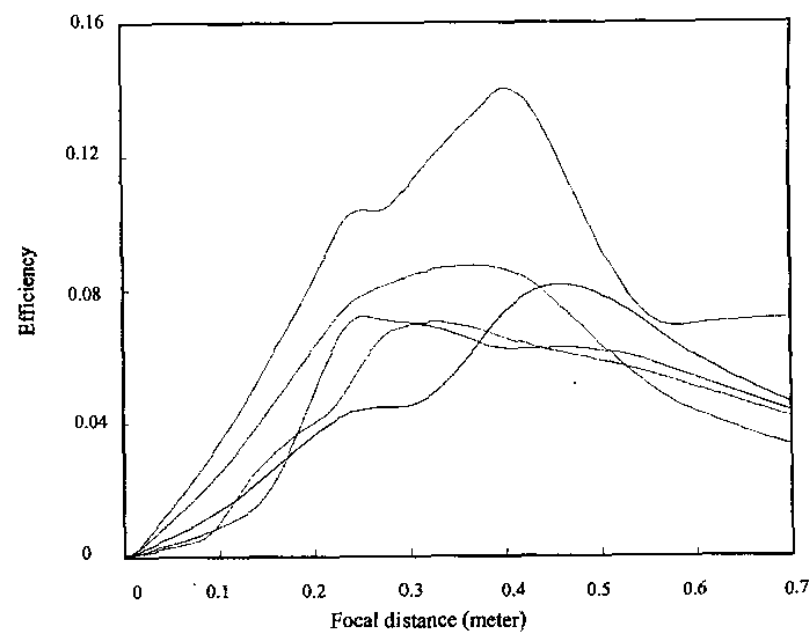

Figure 13. The total efficiency as a function of the focal distance for a feed antenna with $n=65: \cdots \cdots \cdots \cdots \cdot \theta_{\text {scan }}=0^{\circ}$; - - -... $\theta_{\text {scan }}=5^{\circ} ;-\theta_{\text {scan }}=10^{\circ} ;--\cdots \theta_{\text {scan }}=15^{\circ} ;-\cdots-\cdot \theta_{\text {scan }}=20^{\circ}$. 


\subsection{Total Efficiency}

The total efficiency, $\varepsilon_{\text {total }}$, takes into account all effects mentioned above. The total efficiency is defined as

$$
\varepsilon_{\text {total }}=\frac{\text { actual gain }}{\text { ideal gain of an antenna with the same effective area }}
$$

The gain of an ideal antenna is given by

$$
G_{i d e a l}=\left(\pi R^{2}\right)\left(\frac{4 \pi}{\lambda^{2}}\right) \cos ^{2}\left(\theta_{\text {scan }}\right)
$$

Hence, the expression for the total efficiency becomes

$$
\begin{aligned}
& \varepsilon_{\text {total }}= \frac{F^{n}(n+1)\left[\cos ^{2}\left(\theta_{\text {scan }}\right) \cos ^{2}\left(\phi_{\text {scan }}\right)+\sin ^{2}\left(\phi_{\text {scan }}\right)\right]}{2 \pi^{2} R^{2} \cos ^{2}\left(\theta_{\text {scan }}\right)} \\
& \times \mid \int_{0}^{2 \pi} \int_{0}^{R} \frac{r^{\prime} m a s k\left(r^{\prime}, \phi^{\prime}, F, \theta_{\text {scan }}, \phi_{\text {scan }}, \lambda\right)}{\left(F^{2}+r^{\prime 2}\right)^{\frac{1}{4}(n+2)}} \\
&\left.x e^{-j k\left[\sqrt{F^{2}+r^{\prime 2}}-r^{\prime} \sin \left(\theta_{\text {scan }}\right) \cos \left(\phi^{\prime}-\phi\right)\right]} d r^{\prime} d \phi^{\prime}\right|^{2} .
\end{aligned}
$$

A number of simulations has been done to evaluate the performance of the efficiency as a function of elevation scanning angle. The behavior of the efficiency was almost the same. Table 1 shows the parameters used for the simulations. Note that only focused systems were analyzed. Therefore, varying the focal distance means changing the masking to correspond to another focal distance, and moving the feed to this new focal point. Table 2 shows the performance of different efficiencies as a function of elevation scanning angle for two different feed patterns. Figures 12 and 13 show the total efficiency as a function of the focal distance for $\phi_{\text {scan }}=0^{\circ}$ for several values of $\phi_{\text {scan }}$. The antenna has a low efficiency.

It is possible to increase the efficiency of the antenna by including phase-correcting zones, instead of transparent/blocking zones. In this case the zones that add $180^{\circ}$ to the phase of the incoming field replace the blocked zones. This increases the efficiency, since all power incident on the Fresnel-zone plate is effectively used. This corresponds to an area efficiency of $100 \%$. Introducing phase-correcting sub-zones can increase the efficiency even more. These sub-zones have a phase correcting factor of less than $\pi$ radians. This increases the phase efficiency.

\section{Co- and Cross-Polarization}

The two components of the electric far field are given by

$$
\begin{aligned}
& E_{\theta}(\theta, \phi)=\frac{j \sqrt{\eta P_{t} F^{n}(n+1)}}{\sqrt{\pi} \lambda r} e^{-j k r} i n t_{1}, \\
& E_{\phi}(\theta, \phi)=\frac{j \sqrt{\eta P_{t} F^{n}(n+1)}}{\sqrt{\pi} \lambda r} e^{-j k r} \cos (\theta) i n t_{2},
\end{aligned}
$$

where $i n t_{1}$ and $i n t_{2}$ are given by

$$
\begin{aligned}
& i n t_{1}=\int_{0}^{2 \pi} \int_{0}^{R}\left[\left[\frac{\sin (\phi)\left\{F\left[1-\cos ^{2}\left(\phi^{\prime}\right)\right]+\cos ^{2}\left(\phi^{\prime}\right) \sqrt{F^{2}+r^{\prime 2}}\right\}}{\left(F^{2}+r^{\prime 2}\right)^{\frac{1}{4} n+1}}\right.\right. \\
& \left.+\frac{\cos (\phi) \cos \left(\phi^{\prime}\right) \sin \left(\phi^{\prime}\right)\left(F-\sqrt{F^{2}+r^{\prime 2}}\right)}{\left(F^{2}+r^{\prime 2}\right)^{\frac{1}{4} n+1}}\right] \\
& x e^{-j k\left[\sqrt{F^{2}+r^{\prime 2}}-r^{\prime} \sin (\theta) \cos \left(\phi^{\prime}-\phi\right)\right]} \\
& \left.\times r^{\prime} \operatorname{mask}\left(r^{\prime}, \phi^{\prime}, F, \theta_{s c a n}, \phi_{s c a n}, \lambda\right)\right) d r^{\prime} d \phi^{\prime} \\
& \text { int }_{2}=\int_{0}^{2 \pi R} \int_{0}^{R}\left(\left[\frac{\cos (\phi)\left\{F\left[1-\cos ^{2}\left(\phi^{\prime}\right)\right]+\cos ^{2}\left(\phi^{\prime}\right) \sqrt{F^{2}+r^{\prime 2}}\right\}}{\left(F^{2}+r^{\prime 2}\right)^{\frac{1}{4} n+1}}\right.\right. \\
& \left.+\frac{\sin (\phi) \cos \left(\phi^{\prime}\right) \sin \left(\phi^{\prime}\right)\left(\sqrt{F^{2}+r^{\prime 2}}-F\right)}{\left(F^{2}+r^{\prime 2}\right)^{\frac{1}{4} n+1}}\right] \\
& x e^{-j k\left[\sqrt{F^{2}+r^{\prime 2}}-r^{\prime} \sin (\theta) \cos \left(\phi^{\prime}-\phi\right)\right]} \\
& \left.\times r^{\prime} . \operatorname{mask}\left(r^{\prime}, \phi^{\prime}, F, \theta_{s c a n}, \phi_{s c a n}, \lambda\right)\right) d r^{\prime} d \phi^{\prime}
\end{aligned}
$$

The total gain can be calculated from

$$
G_{t o t a l}=\frac{2 \pi r^{2}}{\eta P_{t}}\left(\left|E_{\phi}\right|^{2}+\left|E_{\theta}\right|^{2}\right) .
$$

To calculate the co- and cross-polar gains, Ludwig's third definition of cross-polarization is used [18]:

$$
\begin{aligned}
& E_{\text {co }}(\theta, \phi)=\sin (\phi) E_{\theta}(\theta, \phi)+\cos (\phi) E_{\phi}(\theta, \phi), \\
& E_{\text {cross }}(\theta, \phi)=\cos (\phi) E_{\theta}(\theta, \phi)-\sin (\phi) E_{\phi}(\theta, \phi) .
\end{aligned}
$$

Therefore, the following expressions for the co- and cross-polar gains are obtained:

$G_{c o}=\frac{2 \pi r^{2}}{\eta P_{t}}\left|E_{c o}\right|^{2}=\frac{2 \pi r^{2}}{\eta P_{t}}\left|\sin (\phi) E_{\theta}(\theta, \phi)+\cos (\phi) E_{\phi}(\theta, \phi)\right|^{2}$

$G_{\text {cross }}=\frac{2 \pi r^{2}}{\eta P_{t}}\left|E_{\text {cross }}\right|^{2}=\frac{2 \pi r^{2}}{\eta P_{t}}\left|\cos (\phi) E_{\theta}(\theta, \phi)-\sin (\phi) E_{\phi}(\theta, \phi)\right|^{2}$

Substituting Equations (37) and (38) into Equations (41), (44), and (45), we get

$$
G_{\text {total }}(\theta, \phi)=\frac{2(n+1) F^{n}}{\lambda^{2}}\left(\mid \text { int }\left._{1}\right|^{2}+\mid \cos (\theta) \text { int }\left._{2}\right|^{2}\right),
$$




$$
\begin{gathered}
G_{c o}(\theta, \phi)=\frac{2(n+1) F^{n}}{\lambda^{2}}\left|\sin (\phi) i n t_{1}+\cos (\phi) \cos (\theta) i n t_{2}\right|^{2}, \\
G_{\text {cross }}(\theta, \phi)=\frac{2(n+1) F^{n}}{\lambda^{2}}\left|\cos (\phi) i n t_{1}-\sin (\phi) \cos (\theta) i n t_{2}\right|^{2},
\end{gathered}
$$

where $i n t_{1}$ and int $_{2}$ are given by Equations (39) and (40). Maximum cross polarization is found for $\phi=\phi_{\text {scan }}=45^{\circ}, 135^{\circ}, 225^{\circ}$, and $315^{\circ}$. Therefore, cross-polarization patterns have been plotted for $\phi=\phi_{s c a n}=45^{\circ}$. Figure 14 shows the co- and cross-polarization patterns as a function of elevation angle.

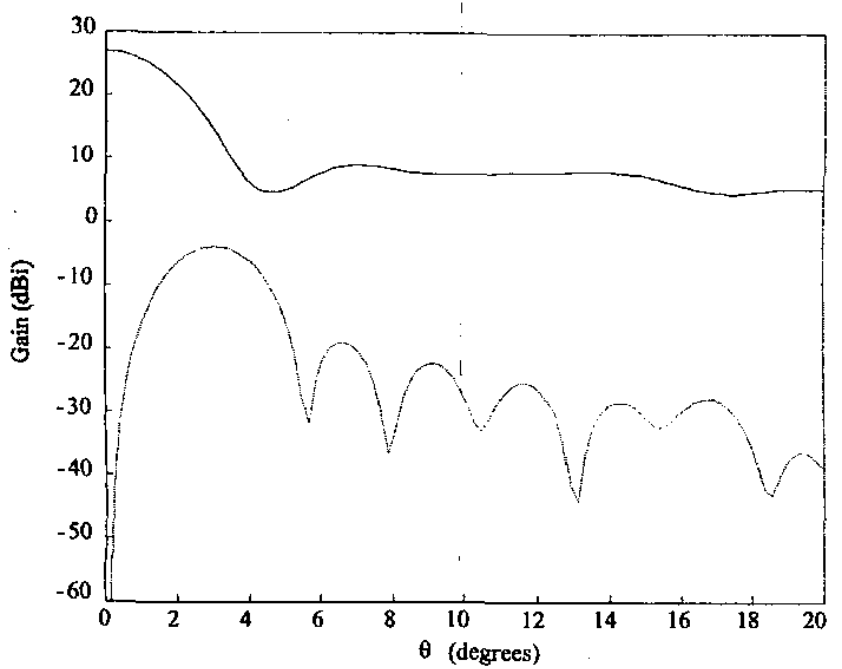

Figure 14a. Co-polarization (solid line) and cross-polarization (dashed line) patterns for $\theta_{\text {scan }}=0^{\circ}, \phi=\phi_{\text {scan }}=45^{\circ}, F=9 \mathrm{~cm}$, $n=5$.

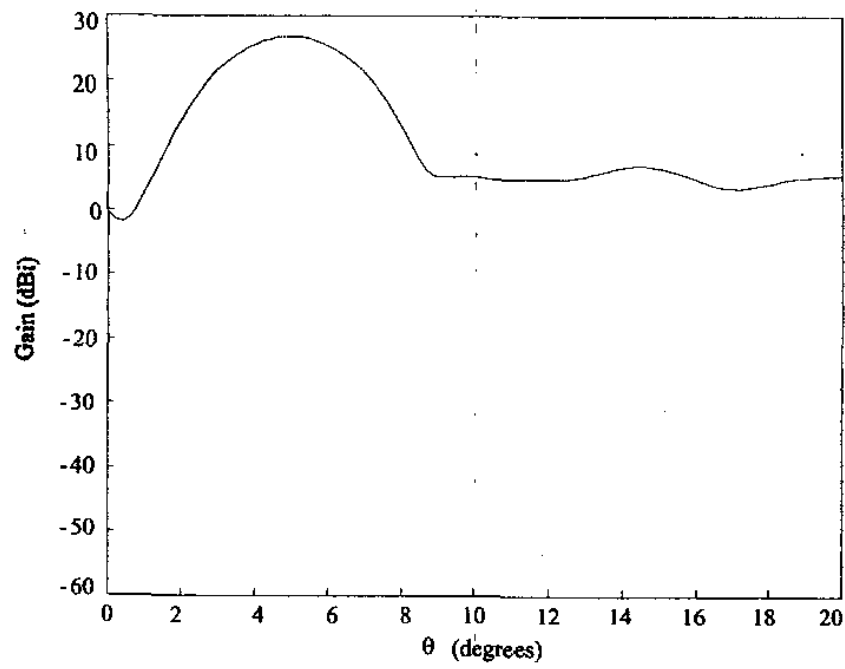

Figure 14b. Co-polarization (solid line) and cross-polarization (dashed line) patterns for $\theta_{\text {scan }}=5^{\circ}, \phi=\phi_{\text {scan }}=45^{\circ}, F=9 \mathrm{~cm}$, $n=5$.

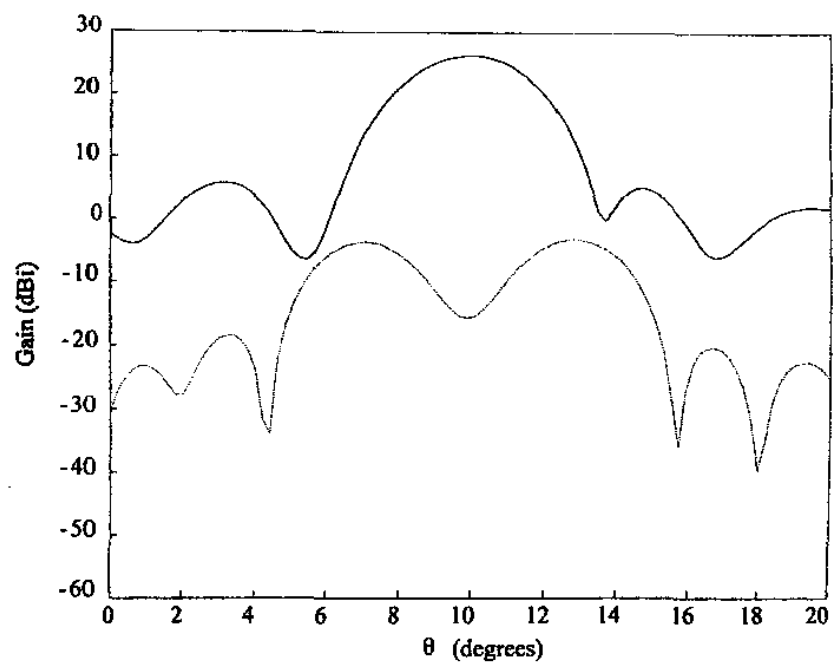

Figure 14c. Co-polarization (solid line) and cross-polarization (dashed line) patterns for $\theta_{\text {scan }}=10^{\circ}, \phi=\phi_{\text {scan }}=45^{\circ}$, $F=9 \mathrm{~cm}, n=5$.

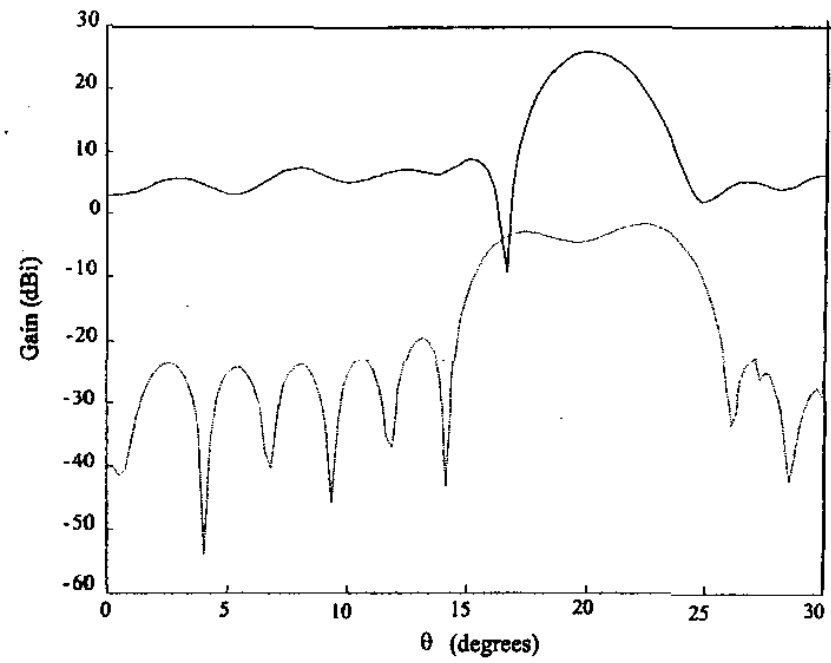

Figure 14d. Co-polarization (solid line) and cross-polarization (dashed line) patterns for $\theta_{\text {scan }}=20^{\circ}, \phi=\phi_{\text {scan }}=45^{\circ}$, $F=9 \mathrm{~cm}, n=5$.

\section{Conclusion}

A beam-scanning antenna has been analyzed and designed at $\mathrm{Ka}$ band. The antenna is based on the principle of the Fresnelzone-plate antenna. The scanning of the beam is achieved by using a laser to project different patterns onto a wafer. The antenna can scan its beam in both the azimuth and the elevation planes. The sidelobe level is lower for an antenna with a smaller focal distance, and increases with increasing scan angle, $\theta_{\text {scan }}$. The beamwidth of the antenna with a wafer diameter $D=20 \mathrm{~cm}$ is approximately $3^{\circ}$. An antenna with a wafer diameter $D=20 \mathrm{~cm}$ and a focal distance $F=40 \mathrm{~cm}$ has a maximum gain of $29.2 \mathrm{~dB}$. For a focal distance of $F=9 \mathrm{~cm}$, the gain decreases to $27.3 \mathrm{~dB}$. The gain decreases with increasing scan angle, proportional to $\cos ^{2}\left(\theta_{\text {scan }}\right)$. The efficiency and cross-polarization of a Fresnel-zone-plate-antenna-based 
beam-scanning antenna were analyzed. It was found that the efficiency of the antenna is very low $(6 \%$ to $14 \%)$. The cross-polarization isolation increases with increasing scan angle, $\theta_{\text {scan }}$ : from $51 \mathrm{~dB}$ for $\theta=\theta_{\text {scan }}=5^{\circ}$ to approximately $30 \mathrm{~dB}$ for $\theta=\theta_{\text {scan }}=20^{\circ}$. Maximum efficiency was found when the edge illumination of the wafer is approximately -10 to $-12 \mathrm{~dB}$. However, no clear optimum value for the edge illumination has been found. The results show serious limitations for the use of the antenna in applications where efficiency is an important factor.

\section{References}

1. M. Hajian, S. H. Heijnen, F. Groen, P. Hakkaart, and L. P. Ligthart, "Modeling and Measuring the Reflection and Transmission of a Silicon Wafer in the X-and Ka-Bands under Illumination of Light in a Closed Waveguide Structure," Microwave and Optical Technology Letters, 31, 5, 2001, pp. 349-353.

2. M. Hajian, W. Keizer, B. J. Reits, and L. P. Ligthart, "Concept of a Scanning Beam Antenna at $35 \mathrm{GHz}$ Based on Photoconductivity Technology," 20th ESTEC Antenna Workshop on MMW Antenna Technology and Antenna Measurement, June 18-20, 1997, ESTEC, Noordwijk, The Netherlands.

3. L. Leyten and M. H. A. J. Herben, "Vectorial Far-Field and Analysis of the Fresnel-zone Plate Antenna: A Comparison with the Parabolic Reflector Antenna," Microwave Optical Technology Letters, 5, 2, February 1992.

4. L. C. J. Baggen and M. H. A. J. Herben, "Design Procedure for a Fresnel Zone Plate Antenna," International Journal of Infrared and Millimeter Waves, 14, 6, 1993, pp. 1341-1352.

5. J. Sluijter, M. H. A. J. Herben, and O. J. G. Vullers, "Experimental Validation of PO/UTD Applied to Fresnel Zone Plate Antennas," Microwave and Optical Technology Letters, 9, 2, June 5, 1995 .

6. D. Hristov, Fresnel Zones in Wireless Link, Zone Plate Lenses and Antennas, Norwood, MA, Artech House, 2000.

7. J. P. Woerdman, "Some Optical and Electrical Properties of a Laser-Generated Free-Carrier Plasma in Si," PhD thesis, December 1971, Technical University Delft, The Netherlands.

8. J. C. Wiltse, "History and Evolution of Fresnel Zone Plate Antennas for Microwaves and Millimeter Waves," IEEE International Symposium on Antennas and Propagation Digest, 2, (Special session on "Status and Future of Fresnel Zone Plate Antennas"), Orlando, FL, July 11-16, 1999, pp. 722-725.

9. J. E. Garrett and J. C. Wiltse, "Fresnel Zone Plate Antennas for Microwaves at Millimeter Wavelengths," International Journal of Infrared and Millimeter Waves, 12, 1991, pp. 195-220.

10. F. Sobel, F. L. Wentworth, and J. C. Wiltse, "Quasi-Optical Surface Waveguide and Other Components for the 100 - to $300 \mathrm{Gc}$ Region," IRE Transactions on Microwave Theory and Techniques, 9, November 1961, pp. 512-518.

11. Y. J. Guo and S. K. Barton, "Offset Fresnel Zone Plate Antennas," International Journal of Satellite Communications, 12, 1994, pp. 381-385.
12. Y. J. Guo, I. H. Sassi, and S. K. Barton, "Multilayer Offset Fresnel Zone Plate Reflector," IEEE Microwave and Guided Wave Letters, 4, June 1994, pp. 196-198.

13. C. A. Balanis, Antenna Theory, Analysis and Design, Second Edition, New York, John Wiley \& Sons.

14. S. Silver, Microwave Antenna Theory and Design, London, Peter Peregrinius Ltd/IEE, 1984.

15. L. C. J. Baggen, C. J. J. Jeronimus, and M. H. A. J. Herben, "The Scan Performance of the Fresnel-Zone Plate Antenna: A Comparison with the Parabolic Reflector Antenna," Microwave and Optical Technology Letters, 6, 13, October 1993

16. L. C. J. Baggen, "The Fresnel Zone Plate Antenna: Design and Analysis," Eindhoven, Netherlands, report of graduation work, December 1992.

17. G. A. de Vree, "Efficiency and Cross-Polarization of Beam Scanning Antenna at Millimeter Wave Band Based on Photoconductivity Using Fresnel Zone Plate Technique," Thesis Report, TU-Delft/IRCTR, June 2002.

18. A. C. Ludwig, "The Definition of Cross Polarization," IEEE Transactions on Antennas and Propagation, January 1973, pp. 116-119.

\section{Introducing the Feature Article Authors}

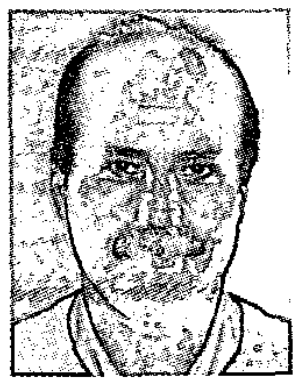

M. Hajian was born in Iran on April 21, 1957. He received his BS in Physics from the University of Oklahoma in the USA in 1981, and the MS degree in Electrical Engineering from Delft University of Technology in 1990. Since 1990, he has been with the IRCTR/Microwave and Radar Laboratory of the Delft University of Technology. In 1995, he became Senior Lecturer, teaching courses in antennas. He was the Netherlands representative of $\mathrm{EC} / \mathrm{COST} 260$ on adaptive antennas. His major interests are antennas and propagation, antenna near-field measurement techniques, antenna modeling, antenna signal processing, smart antennas, and space-time coding in mobile communication systems.

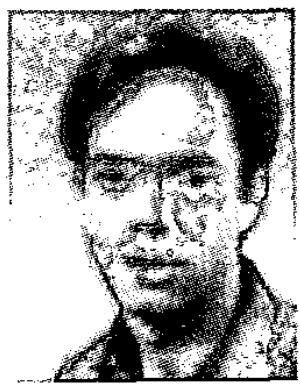

Gerralt de Vree was born on May 9, 1977, in Zwolle, The Netherlands. In 2002, he received his MSc degree in Electrical 
Engineering at TU-Delft in The Netherlands. His major interests are telecommunications and microwave engineering.

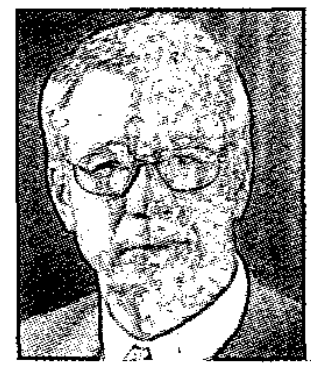

L. P. Ligthart was born in Rotterdam on September 15 1946. He graduated with distinction in 1969 and received the MS in Electrical Engineering from Delft University of Technology. Since 1969, he has been with the Microwave Laboratory of the Delft University of Technology. In 1974, he became a Senior Lecturer, teaching undergraduate courses in transmission line theory and antennas and propagation. From 1976 to 1977, he spent one year as a Senior Scientist at Chalmers University, Gothenburg, in Sweden. In 1985, he received the $\mathrm{PhD}$ in Technical Sciences, based on his contributions to the design of miniaturized waveguide radiating elements. Prof. Ligthart is Director of IRCTR, covering activities on antennas, propagation, radar, mobile and satellite communication, remote sensing, and electromagnetic compatibility. His present interests include antennas and propagation, radar, and remote sensing. He received the vederprijs-award in 1981, the IEE Blumlein-Brown-Willams Premium award in 1982, and a Doctor Honoris Causa from Moscow State Technical University of Civil Aviation in 1999. He is a Fellow of the IEE and IEEE, Netherlands representative of EC/COST 260 on adaptive antennas and $\mathrm{EC} / \mathrm{COST}$ on advanced weather radar. He has published over 152 scientific papers. Ati

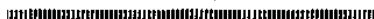

\section{Editor's Comments Continued from page 8}

The Antenna Designer's Notebook, edited by Tom Milligan, has two contributions this time. The first, by J. C. Brégains, F. Ares, and E. Moreno, considers circular aperture distributions that generate azimuthally symmetric patterns. The authors look at how the power pattern degrades as a function of measurement distance from the aperture - over distances ranging from the near field to the far field - for both real and complex continuous Taylor distributions in the aperture. The results show some potentially important effects of the measurement distance, including the fact that at near-field distances, the degradation in pattern in terms of sidelobe level and ripple is less for real distributions than for complex distributions. The second Antenna Designer's Notebook contribution is by Simsek Demir, and it considers the design of wideband impedance-matching transformers. What is particularly useful about this contribution is that it deals with the real-world limitations that exist on the available impedance levels of a transformer.

Can a patent application be simplified by incorporating material from other sources by reference? That is the question Lee Henderson answers in his Intellectual Property and Patent Abstracts column. There is a short answer, and a long answer, and you need to understand the long answer if you're contributing to the preparation of a patent application.

In the PACE Report, edited by Michael Johnson, the recent theme of developing engineers who are also entrepreneurs contin- ues. The contribution, by Vijay K. Arora and Lorenzo Faraone, puts forward the proposition that universities must foster the development of such engineer-entrepreneurs as an inherent part of their curricula if the universities - and the engineers they produce - are to be successful in the 21 st century.

What is known as the hybrid model for propagation loss in a coal-mine tunnel consists of a free-space model for propagation up to certain distances, and a waveguide model for propagation over longer distances. Each model has a different value for the propagation constant. While the free-space propagation constant can be determined fairly readily, the best value for the propagation constant for the waveguide model varies with the tunnel's specific attenuation factor (based on measurements), and the distance at which the crossover in using the two models occurs. In the Wireless Corner, edited by Tuli Herscovici and Christos Christodoulou, Bazil Taha Ahmed, Miguel Calvo Ramón, and Leandro de Haro Ariet study how the models behave as a function of these various parameters. The results show that some values of the propagation constant used in the past for the waveguide model are reasonable for certain ranges of the parameters, but that other values could be more appropriate for different ranges of the parameters.

\section{Thank You to Ernie Smith}

Ernie Smith has been an important contributor to the Magazine since it became a magazine. His Propagation Corner, and his solicitation of feature articles dealing with propagation, have played an important role in keeping the "P" in the "A\&P" Magazine. I hope you will join me in thanking Emie for his more than 13 years of outstanding service to our Society in this capacity. Personally, his wise council and friendship have been invaluable. The good news is that although he will probably be making fewer technical contributions as he indicated in his column in the August issue, he has agreed to still provide us with news of the propagation community from time to time.

\section{We Welcome Bodo Reinisch}

I am very pleased to announce that Bodo Reinisch has agreed to join the Magazine staff in the role of Associate Editor for Propagation. In this role, he will support the "propagation" content of the Magazine, and take over primary responsibility for the Propagation Corner. Please welcome him, and offer him your help in keeping our Society informed of what is going on in the propagation field. Bodo is perhaps best known for the development of the global Digisonde network, which provides real-time electrondensity profiles and other ionospheric characteristics worldwide, now via the Internet. More information can be found in the Propagation Corner in this issue.

\section{Solutions for a Pentium 4 Bug and Other Problems with the Digital Archive}

Some users have reported problems with the ASTAware search engine used in the AP-S Digital Archive. According to Parity Computing, the company that helped to prepare the Digital Archive, there apparently is a problem with the version of Java that Continued on page 45 